Proceedings of the ASME 2010 International Design Engineering Technical Conferences \&
Computers and Information in Engineering Conference
IDETC/CIE 2010
August 15-18, 2010, Montreal, Quebec, Canada

DETC2010-28889

\title{
A SYSTEMATIC METHODOLOGY FOR ACCURATE DESIGN-STAGE ESTIMATION OF ENERGY CONSUMPTION FOR INJECTION MOLDED PARTS
}

\author{
Alexander Weissman \\ Department of Mechanical Engineering \\ University of Maryland \\ College Park, Maryland \\ Satyandra K. Gupta \\ Department of Mechanical Engineering and \\ Institute for Systems Research \\ University of Maryland \\ College Park, Maryland
}

\author{
Arvind Ananthanarayanan \\ Department of Mechanical Engineering \\ University of Maryland \\ College Park, Maryland \\ Ram D. Sriram \\ Manufacturing Systems Integration Division \\ National Institute of Standards and Technology \\ Gaithersburg, MD
}

\begin{abstract}
Today's ubiquitous use of plastics in product design and manufacturing presents significant environmental and human health challenges. Injection molding, one of the most commonly used processes for making plastic products, consumes a significant amount of energy. A methodology for accurately estimating the energy consumed to injection-mold a part would enable environmentally conscious decision making during the product design. Unfortunately, only limited information is available at the design stage. Therefore, accurately estimating energy consumption before the part has gone into production can be challenging. In this paper, we describe a methodology for energy estimation that works with the limited amount of data available during the design stage, namely the CAD model of the part, the material name, and the production requirements. This methodology uses this data to estimate the parameters of the runner system and an appropriately sized molding machine. It then uses these estimates to compute the machine setup time and the cycle time required for the injection molding operation. This is done by appropriately abstracting information available from the mold flow simulation tools and analytical models that are traditionally used during the manufacturing stage. These times are then multiplied by the power consumed by the appropriately sized machine during each stage of the molding cycle to compute the estimated energy consumption per part.
\end{abstract}

\section{INTRODUCTION}

Over the past several decades, plastics have moved from small-scale application in highly specialized niche markets, to a ubiquitous presence in everyday consumer products. Plastics are popular engineering materials because of their versatility, durability, and relatively low cost. However, they also present significant environmental and human health challenges: they are slow to break down in landfills and oceans, their processing consumes a large amount of energy, and they can release a number of substances during usage and disposal which may have adverse effects on humans and the environment.

One of the most heavily used processes for creating plastic parts is injection molding. In this process, liquefied polymer is injected at high pressure into a mold cavity. The polymer takes the shape of the cavity, and is cooled either passively or actively using water channels. The resulting part is then ejected from the mold cavity, and the molding machine is reset for the next part.

The main environmental concerns associated with injection molding are energy consumption and waste generation. During injection molding, energy is consumed to melt, inject and pressurize the resin, open and close the mold, and pump water for cooling. This energy consumption has significant environmental consequences. In the very countries with the largest injection molding industries, electrical energy is mostly produced through combustion of fossil fuels [1, 2]. The burning of fossil fuels for electricity generation is the largest single anthropogenic source of the greenhouse gas emissions responsible for global warming [3]. Waste, in the form of the additional polymer in sprues and runners, is also another significant environmental concern. This waste is more prominent in injection molds having cold runners. This is because the polymer in the runners in such molds is not part of the final part. This waste is often recycled by regrinding the 
waste polymer into pellets. This, in turn, increases the overall energy consumption. It is therefore clear that, to mitigate the environmental impact of such processes, there is a need for an accurate method for estimating the energy consumption, resource consumption, waste and emissions that result from the plastic manufacturing. Furthermore, it is necessary to look beyond simply the impact of the injection molding facility. Both upstream and downstream impacts accrued during resource extraction, shipping, usage, and disposal should be considered as well. However, this paper does not attempt to perform the entire life cycle assessment for plastic parts. Instead, our scope is limited to the energy consumed during the injection molding operation.

Currently most injection molded parts are optimized at the design stage with respect to the cost and part quality. Once the design has been optimized for these criteria, the mold for part production is machined. This mold could be used for actively measuring the energy consumption by connecting an energy meter to the injection molding machine during production. However, building a typical production-quality mold for energy estimation alone is not economically viable [4]. In order for the energy estimation to be beneficial, it is necessary for the designer to obtain this information at the design stage, before the mold has been machined. The designer can then use this information to optimize the design for energy consumption.

During the design stage, fully characterizing the manufacturing stage is extremely challenging as there are many different factors and parameters which drive energy consumption. These parameters are more than simply the part volume and material choice, which are typically the sole basis on which energy consumption is estimated today. In addition to the volume, other information from the geometry model of the part such as projected area, part depth, and maximum wall thickness has a significant effect on the energy consumption.

The system of runners that carries the molten polymer from the injection nozzle to various cavities in the mold also plays a major role in estimating energy consumption. In some cases, the volume of the runner system can be as large as, or larger than the volume of the part itself. Therefore, significant energy is expended to melt this additional material. The size and arrangement of the runners may also require a larger injection molding machine. Different injection molding machines consume vastly different amounts of energy, based on the size of their clamping mechanisms, screw, heater, and pumps. Production requirements also have an indirect contribution to the energy consumption. For example, production in smaller batches requires that the machine be warmed up and calibrated more often, thus requiring more energy each time a batch is started. Production requirements may also play a role in determining the runner layout of the part, as well as the size of the machine that will be used. Thus, the geometric model of the part, the runner system for the mold, the size of the machine, and the production requirements interact in a complex way to influence the per-part energy consumption during injection molding.

Unfortunately, most of this information is not available during the design stage. Typically, the available data consists of the CAD model of the part, the material to be used, and the production volume. This information can be used to obtain an accurate estimate of energy consumption, but it requires additional simulation tools such as Moldflow and discrete event simulation (DES), and careful application of various analytical models. In addition, non part-specific information, such as a database of runner layouts, and a database of power consumption profiles for various injection molding machines are needed to make appropriate inferences about new part designs. Much of this information is currently either unavailable, or not compiled into an easily accessible database. Therefore, appropriate templates must be constructed for gathering and organizing this information in a systematic manner.

In this paper, we propose a methodology to estimate the per-part energy requirement for injection molded parts during the design stage. This methodology begins by utilizing the information provided by the CAD model of the part and information on similar parts which have already been molded. From this information, the material parameters, and the production volume, inferences are made to calculate the parameters of a surrogate runner system, a surrogate injection molding machine, and the production policy for manufacturing the part. From these estimated parameters, we compute the time spent during each stage of setup and molding. This is done by appropriately abstracting information from the mold flow simulation tools and analytical models that are traditionally used during the design stage. Next, information on the runner layout of similar parts, and the power consumption profile of an appropriately sized injection molding machine is collected. Finally, the total energy consumption in kilojoules per part is computed by multiplying the power consumed by the machine in each stage of molding, and then multiplying it by the estimated part-specific setup and cycle times.

\section{OVERVIEW OF EXISTING METHODS}

Currently, several heuristics exist for assessing the environmental and health impact of a given product, process, or system. One such method is known as Life Cycle Assessment (LCA), and is defined in the ISO 14040 standard. According to ISO 14040, LCA consists of four stages: 1) goal and scope, 2) inventory, 3) assessment, and 4) interpretation. In the "goal and scope" stage, the problem and its boundaries are defined. In the "inventory" stage, the materials used, processes executed, and waste produced at each stage of the product's life cycle are quantified. In the "assessment” stage, values from the LCA are used to calculate, normalize, and weight the impact of the product in one or more categories. Various assessment methodologies [5] have been developed which can be adapted with LCA software such as $\mathrm{GaBi}$ [6] or SimaPro [7]. In the final stage, “interpretation”, the reviewer interprets the results 
of the assessment, draws conclusions, and makes recommendations.

The "goal and scope" and "interpretation" stages require qualitative and context-specific judgement, and thus intrinsically require a human thinker. Therefore, there is little scope for improvement in this stage from an engineering standpoint. The "assessment" stage is fairly well supported by current generation LCA tools. Sophisticated algorithms have been developed to transform input data such as greenhouse gas emissions, water pollutants, and raw materials extracted from nature, into measurable impacts on ecosystems, climate change, and human health. Currently, the weakest point of LCA is the "inventory" stage, where the input data is calculated and tabulated.

For injection molded parts, a proper LCA requires that the energy consumed during manufacturing be accounted for. Energy consumed at other stages of the plastic life cycle such as petroleum refining, shipping, usage, and recycling must also be considered, but accounting for this data will be considered in future work. Current LCA models of energy consumption for the injection-molding process use an allocation scheme, based on specific energy consumption (SEC) [8]. SEC is defined as the amount of energy used by a specific process for a unit quantity of material. The mass of the part, which can be obtained from a CAD model, is multiplied by the injectionmolding SEC for the given material, which can be found in an LCA database. From this calculation, an estimate of energy consumption is obtained. This process is shown in FIGURE 1.

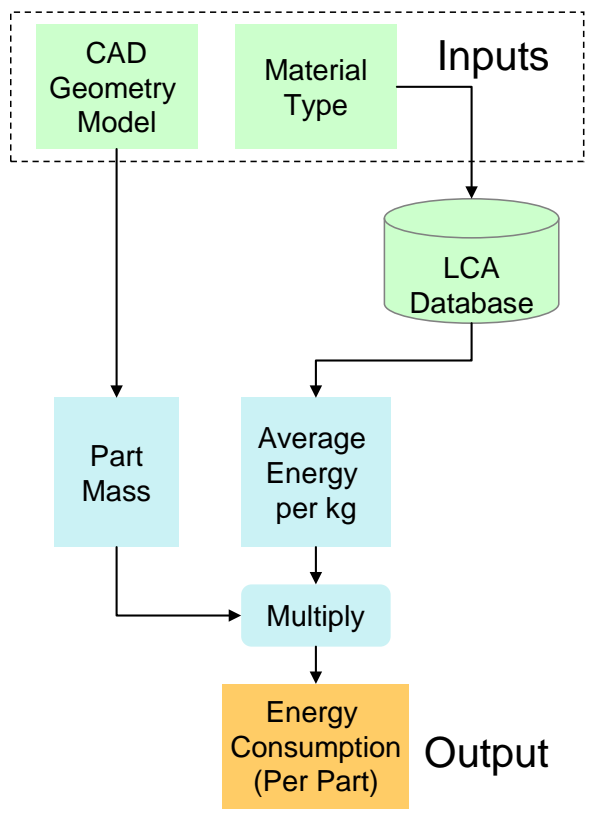

FIGURE 1: CURRENT METHOD USED TO INVENTORY ENERGY CONSUMPTION FOR INJECTION MOLDING.

Unfortunately, the available LCA databases only provide an average over the range of machines used in the industry. This is inadequate because properties of the specific machine used dramatically influence energy consumption.
Larger machines require more thermal energy to maintain the polymer temperature, and more power to move the heavier injection and clamping mechanisms. These generalizations lead to wildly inaccurate energy estimates.

In addition, the allocation scheme based on SEC and part mass do not account for the influence of part geometry and cycle time. Parts having the same volume and therefore the same mass, but different geometry can have significantly different cycle times and therefore require different amounts of energy to manufacture. For example, let us consider the two parts shown in FIGURE 2. Both parts are made using the same material and have the same volume and mass. However, the maximum wall thickness of the smaller, more compact part (a) is twice that of the larger, thinner part (b). The cooling time for an injection molded part is proportional to the square of the maximum wall thickness [4]. Therefore the cooling time for the cup in FIGURE 2 (a) will be approximately 4 times that of the cup in FIGURE 2 (b). During the cooling time, the machine continues to idle and consume energy. Therefore increased cooling time, along with increasing the cycle time of the operation, also results in increased energy consumption. Studies by Gutowski [9] and Krishnan [10, 11] show that the energy consumed by overhead operations such as maintaining the polymer melt and the mold temperature along with pumping fluids and coolants, can be more than the energy used during each production run. Thick parts may especially require active cooling, which requires use of even more energy to supply coolants.

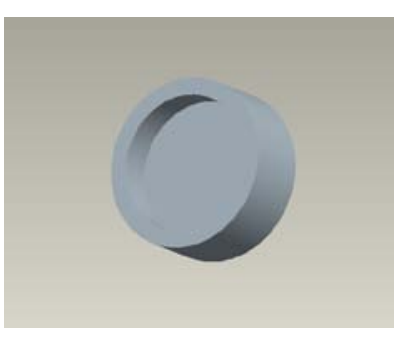

(a)

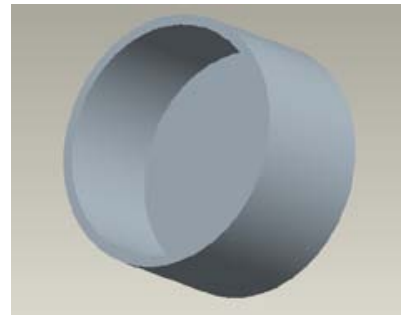

(b)
FIGURE 2: TWO DIFFERENT PARTS WITH EQUAL VOLUME BUT DIFFERENT WALL THICKNESSES AND COOLING TIMES. PART (A) HAS A WALL THICKNESS OF 0.05 IN., WHILE PART (B) HAS A WALL THICKNESS OF 0.025 IN. BOTH PARTS HAVE A VOLUME OF 3.34 IN $^{3}$.

Gutowski and Krishnan [9-11] have shown that machines with a typically higher throughput tend to consume less energy per part. This can be explained by the influence that cycle time has on energy consumption as described above. Since the baseline idling energy is relatively constant, a machine having lower typical cycle times allocates less idling energy per part.

To account for the effects of baseline idling energy, Gutowski divides the specific energy consumption into two components: one component represents the energy used while 
the machine is idling, and the second component represents the additional energy used to process each unit of material. However, this method still does not account for the variations in power consumption at different stages of the molding cycle. A 2007 study [12] investigating the effects of conformal cooling channels on energy consumption showed that a $40 \%$ reduction in cycle time for the same part on the same machine results in only a $20 \%$ reduction in energy consumption. This suggests that the portion of the cycle that was shortened consumed power at a rate lower than the average for the entire molding cycle. Therefore, an approach that accounts for a specific part geometry and machine at each stage of the molding cycle could help to achieve a more accurate estimate of energy consumption.

\section{PROBLEM STATEMENT}

The goal of our paper is to develop a methodology for estimating the energy required to manufacture a part during the design stage. This will enable designers to make changes to the design that minimize the overall energy consumption. Unfortunately, as mentioned earlier, only limited information is available to estimate molding energy consumption at the design stage. Typically, information is available from three sources: the design team, the material supplier, and industry databases. From these sources, the following set of data can be obtained which comprises the inputs to our methodology.

\section{Information available from design team}

(1) A geometric model of the part. This consists of a model created in a common CAD package such as AutoCAD, ProEngineer, or SolidWorks. This model can be used for determining volume, part depth, maximum wall thickness, and projected area.

(2) Material. The precise material must be known, including the material manufacturer, resin type, and filler type and concentration.

(3) Part delivery schedule. To predict the energy consumed during setup and maintenance of the injection molding machine, it is necessary to determine how often setups and maintenance will be performed. This depends on how often the machine is run to produce a batch of parts, which we will call the batch period. The batch period depends primarily on the delivery schedule required by the customer, but can be optimized using warehouse storage to minimize cost. To determine the optimal batch period, we must know the delivery schedule, which consists of the following pieces of information:

a. Delivery volume. This is the number of parts that must be delivered at a time to the customer.

b. Delivery period. This is the interval between deliveries of parts to the customer, measured in days.

c. Production volume. This is the total number of parts that the customer needs. We assume that this is a whole multiple of the delivery volume.

\section{Material Information}

Data on molding parameters for the material can be procured from material datasheets provided by suppliers. For the selected material, the following information is required:

(1) Density. This is the density of the molded material, in $\mathrm{g} / \mathrm{cm}^{3}$.

(2) Specific heat capacity. This is the energy required to heat one gram of the material by one degree Celsius. Units are $\mathrm{J} / \mathrm{g}-{ }^{\circ} \mathrm{C}$.

(3) Recommended injection pressure. This is the maximum pressure at the nozzle during the filling phase. Units are $\mathrm{N} / \mathrm{cm}^{2}$.

(4) Recommended polymer injection temperature. This is the temperature at which the polymer is injected into the cavity. Units are degrees Celsius.

(5) Recommended mold temperature. This is the recommended temperature to which the mold should be heated prior to injection. Units are degrees Celsius.

(6) Recommended ejection temperature. This is the recommended temperature to which the molded part should be cooled prior to ejection from the mold. Units are degrees Celsius.

\section{Database Construction}

In addition, we can use information from industry databases that most closely matches our anticipated manufacturing scenario. For the purposes of this paper, we will construct our own preliminary databases which will be expanded in the future. The information in these databases consists of the following:

(1) Machine database. This database contains comprehensive information on a set of injection molding machines of varying sizes. For each machine, the following information must be available:

a. Clamping force. This is the maximum force that the clamping mechanism is able to apply to the exterior of the mold to counter the pressure exerted by the flow of polymer into the mold cavity. Units for this are newtons $(\mathrm{N})$.

b. Shot size. This is the largest volume of polymer that the machine can deliver to the mold cavity in a single cycle. This has units of $\mathrm{cm}^{3}$.

c. Stroke length. This is the maximum possible displacement of the mold from the closed state. This has units of centimeters (cm).

d. Maximum flow rate. This is the maximum rate at which the machine can deliver material through the injection nozzle. Units for this are $\mathrm{cm}^{3} / \mathrm{s}$.

e. Power profile. This is a set of data which provides the average amount of energy used by the machine per 
unit time, during each phase of the machine cycle. In addition to the power required during filling, cooling, and resetting, this profile should also include the average power used during setup, maintenance, and other events during which the machine is idling and thus consuming energy.

f. Dry cycle time. This is the time required for the machine to complete an injection cycle when injecting a standard cavity with air, instead of molten plastic. Units for this are seconds.

g. Average setup time. This is the average time required to setup the machine. Units are measured in seconds.

h. Average number of calibration parts. This is the average number of parts that are discarded during calibration of the machine.

(2) Runner system database. This database must contain many parts with different geometries, quality requirements, number of cavities, and runner systems. The runner system for a new part can be inferred from the runner system of previously manufactured parts with similar geometries, quality requirements, and number of cavities.

Based on the above described information, we seek to estimate the per-part energy consumption for a molded part. This includes the energy used during the molding cycle, as well as the energy used during setup and calibration, amortized over the total number of parts in the batch. We assume that we are dealing with very high production volumes, and thus the energy consumption of making the mold would be very small in comparison and can be ignored. Furthermore, we ignore the energy consumption for machine maintenance in this paper.

\section{OVERVIEW OF APPROACH}

To develop an accurate method for estimating energy consumption for injection molded parts, we have formulated an algorithm consisting of five steps. These steps are:

(1) Determine a surrogate runner arrangement, and its volume, for the mold.

(2) Approximate the parameters of the machine that will be used based on the production requirements.

(3) Estimate various components of the cycle time for molding a part.

(4) Estimate the number of setup operations based on the delivery schedule.

(5) Multiply these times by the appropriate average power used in each stage by the selected machine, and sum to get the total energy consumption.

This approach is summarized in FIGURE 3. First, we analyze the CAD model of our part to determine the mold cavity volume. In addition to the volume of the part, we must also consider the volume of the runner system and sprue. In some parts, especially parts at the small scale, the runner system can be much larger than the part. Hence it is important to carefully select the runner layout for estimating the projected volume of the mold cavity.

\section{SELECTION OF RUNNER LAYOUT}

To arrive at a good estimate of the per-part energy consumption, we must be able to accurately predict how the mold cavities and runner system will be laid out when the part goes into production. Selection of the appropriate runner layout is one of the most challenging problems encountered by mold designers [13]. The problem involves concurrent optimization for 1) ensuring complete filling of the cavities, 2) minimizing the ratio of runner volume to part volume to minimize material waste, and 3) maintaining part quality by ensuring that part quality parameters such as shrinkage, warpage, residual stresses, shear variations etc. are within the specified tolerances.

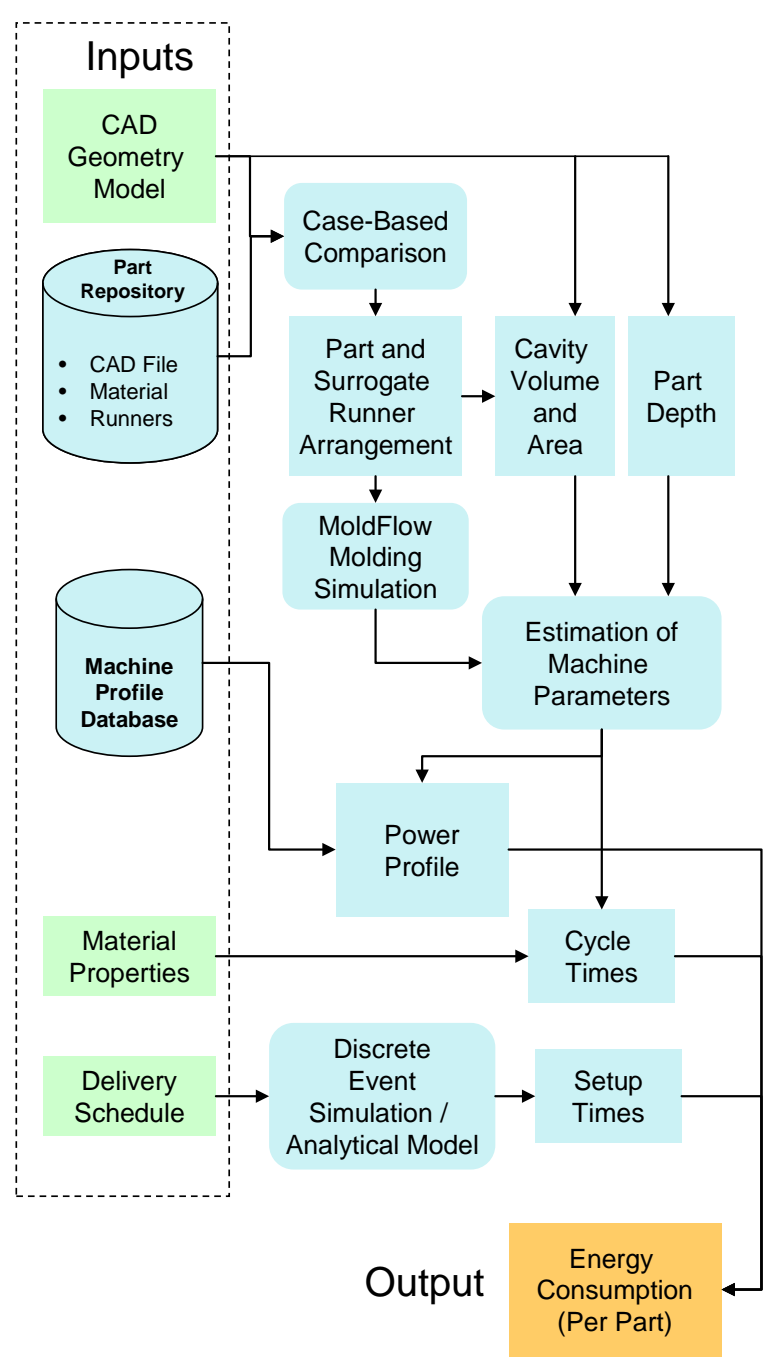

FIGURE 3: GENERAL APPROACH FOR ESTIMATING PER-PART ENERGY CONSUMPTION. 
Considering the above optimization parameters, manufacturers are always looking to maximize the number of cavities in each mold. This strategy increases productivity by reducing the cycle time per part while maintaining the cycle time for each injection. However multiple cavity molds make production of identical parts challenging. This is because there may be discrepancies in the parts in each cavity of the mold depending on the layout of the runner and each cavity in the mold. This discrepancy is caused by several factors as illustrated in FIGURE 4 [14]. Several researchers have studied the effects of discrepancies based on various parameters such as geometric balancing [15], pressure and temperature [16], shrinkage [17], weld-line positioning [18], and total fill time [19]. These discrepancies become even more pronounced as the cavities move further away from the center of the mold. This is because the mold deformation during the packing phase is at a maximum near the center of the mold [13]. Hence as the cavities are moved further away from the center, there is significant difference in the pressures seen in each cavity. This in turn influences the part quality. Hence the parts produced in each cavity are not identical. Researchers have argued that this discrepancy is more pronounced in cavities with eight or more cavities per mold [13]. Hence for the sake of this effort, we will restrict ourselves to molds having up to four cavities.

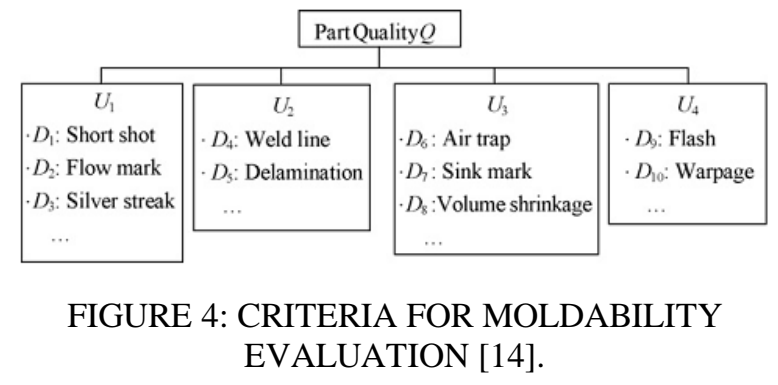

FIGURE 5 illustrates eight different sprue/runner layouts for four-cavity molds. These layouts are commonly used layouts which use fishbone and ladder layouts. The most appropriate runner layout is selected based on the critical quality metrics such as shrinkage, shear level, part density, mold machining constraints etc. while optimizing for the cycle time and the overall runner volume. The geometry of the mold and the sprue location also plays a significant role in the selection of the most appropriate runner layout. Considering the complex nature of this problem, manufacturers currently select the most appropriate runner/sprue layout based on their prior experience. Hence for the purpose of total energy estimation which is the focus of this paper, we will choose the runner design based on our previous injection molding experience. As part of the future work, we will develop a performance heuristic based method to automate the selection of the optimum runner/sprue layout for any given part which is envisaged to be manufactured using injection molding.

In this paper, we will use the part shown in Error! Reference source not found. as a running example. This is a generic housing for an electronic device, and is meant to represent the typical shapes and features found in plastic housings. We have selected Hival ABS HG6 Natural, produced by Ashland Distribution [20] as the material for this part. ABS is a common and widely used plastic for electronic device housings. For our delivery schedule, we assume that the customer requires a shipment of 50,000 parts every two weeks, for a total production volume of 2 million parts. For selecting the runner design for this part we identified a similar part from our injection molding part library. This part uses a four-cavity mold with the runner design illustrated in FIGURE 7. This layout provided for 1) geometric balancing for filling, 2) equal cavity distance from mold center and 3) minimum volume of the runner. Hence, owing to part similarity, we used the same runner design for estimating the energy consumption for molding the example part shown in Error! Reference source not found. This runner design is illustrated in FIGURE 8. We computed the minimum allowable runner size based on filling simulations performed using Moldflow [21]. Finally, we selected the runner diameter based on the tooling restrictions for machining the mold.

Once we selected the runner/sprue layout and the total number of cavities in the mold, we could compute the projected area of the runner system and the runner volume. This information is then used for selecting the machine for completing the injection molding operation.

\section{SELECTION OF MACHINE}

The next step is to estimate the size of the injection molding machine required to mold the part. Machine size is primarily driven by the clamping force required to hold the mold closed during the injection cycle, the shot size required by the volume of the part and runners, and the stroke length required to clear the maximum depth of the part during part ejection [4]. The part volume and maximum depth of the part can be determined from the geometric model. The required clamping force can then be determined from the relationship between the maximum cavity pressure and the projected area of the cavity.

The maximum pressure in the mold can be determined using Moldflow, given the predicted mold design from the first step and the recommended injection pressure. We then assume that the manufacturer will use the cheapest machine which can provide the necessary clamping force, shot size, and stroke length. The required shot size is equal to the volume of the part, plus the volume of the runners and sprue. This total volume can be determined using Moldflow. The stroke length $L_{S}$ is typically estimated by a linear relationship with the maximum depth of the part. A machine which meets these criteria can be looked up in machine database [22]. For this study, we have built a small database of machines based on the list given in [4]. 


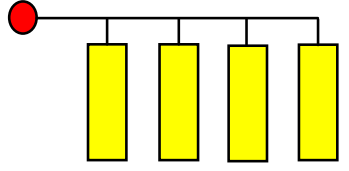

(a) One-sided Ladder

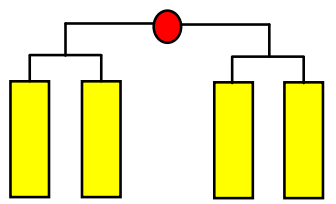

(c) Geometrically balanced twosided Ladder

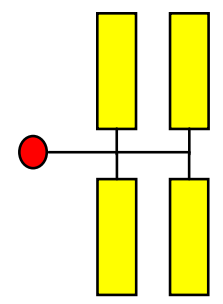

(e) One-sided Fishbone

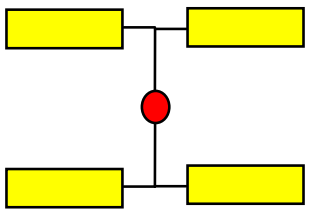

(g) Geometrically balanced two-sided Fishbone

FIGURE 5: DIFFERENT SPRUE AND RUNNER LAYOUT FOR FOUR-CAVITY MOLDS. THE RED CIRCLES REPRESENT THE SPRUE, AND EACH YELLOW RECTANGLE REPRESENTS A SINGLE MOLD CAVITY.
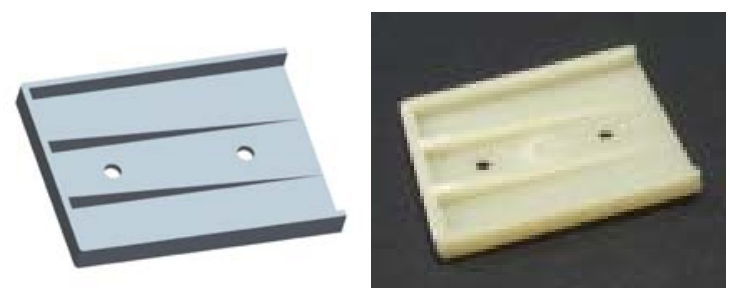

FIGURE 6: CAD MODEL AND MANUFACTURED PRODUCT FOR AN EXAMPLE PART REPRESENTING A GENERIC ELECTRONICS HOUSING.

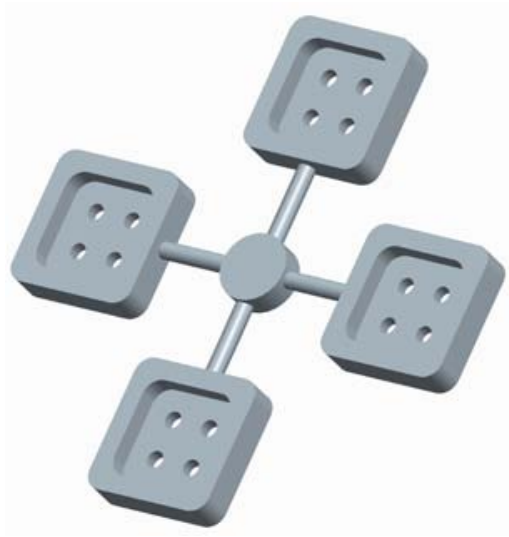

FIGURE 7: RUNNER DESIGN FOR INJECTION MOLDING OF PART IN REPOSITORY AT THE ADVANCED MANUFACTURING LAB.

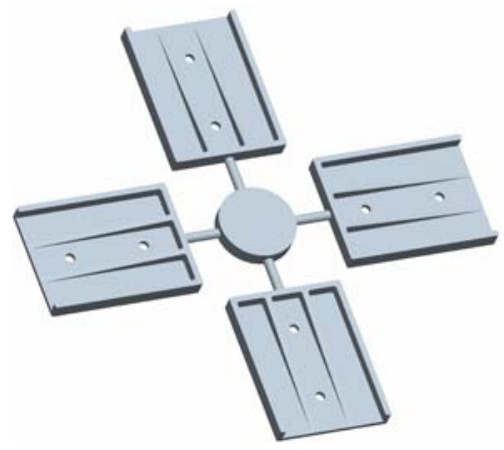

FIGURE 8: RUNNER DESIGN FOR ENERGY ESTIMATION STUDY PART.

Thus, machine selection consists of the following algorithm: Inputs:

$V_{\text {caviy }} \quad$ volume of cavity (shot size), $\mathrm{cm}^{3}$

$P_{\max } \quad$ maximum cavity pressure, $\left(\mathrm{N} / \mathrm{cm}^{2}\right)$

$A_{\text {cavity }} \quad$ projected area of mold cavity parallel to parting line, $\mathrm{cm}^{2}$

$L_{\text {stroke }} \quad$ maximum required stroke length for machine, $\mathrm{cm}$

D maximum part depth, cm

$n_{\text {cavities }} \quad$ number of mold cavities (parts per shot)

\section{Output:}

The selected machine $M$ for the part.

Algorithm selectMachine:

- Compute $F_{\text {clamp }}$ and $L_{\text {stroke }}$.

○ $\quad F_{\text {clamp }}=P_{\max } A_{\text {cavity }}$ 
o $\quad L_{\text {stroke }}=2 D+5$

- Select a machine $M$ from the database of machines for which

o the maximum clamping force $F_{\text {clamp }}^{\max }$ is greater than

$$
F_{\text {clamp }} \text { AND }
$$

o the maximum stroke length $L_{\text {stroke }}^{\max }$ is greater than

$$
L_{\text {stroke }} \text { AND }
$$

o the maximum shot volume $V_{\text {max }}$ is greater than

$$
V_{\text {cavity }} \text { AND }
$$

O the machine rate $c_{\text {machine }}$, in dollars per hour, is minimized.

The geometric attributes for a four-cavity mold for our example part (shown in FIGURE 8) are as follows:

$$
\begin{aligned}
& V_{\text {cavity }}=n_{\text {cavities }} V_{\text {part }}+V_{\text {runners }} \\
& V_{\text {cavity }}=4 * 4.500 \mathrm{~cm}^{3}+2.870 \mathrm{~cm}^{3}=20.87 \mathrm{~cm}^{3} \\
& A_{\text {cavity }}=84.10 \mathrm{~cm}^{2} \\
& D=0.5398 \mathrm{~cm} \\
& h_{\max }=0.2874 \mathrm{~cm}
\end{aligned}
$$

The maximum pressure in the cavity is estimated as $50 \%$ of the recommended injection pressure for the selected material [4]. For Hival ABS HG6 Natural, this gives us the maximum cavity pressure as:

$$
P_{\text {max }}=5 \mathrm{kN} / \mathrm{cm}^{2}
$$

This estimated value is verified using MoldFlow simulations of the cavity filling stage with the selected machine and material parameters. If a discrepancy is found, then this value is modified using MoldFlow simulation data.

Given these values, we can compute the required clamping force and stroke length to successfully mold the part. We can then select the machine from our database that minimizes cost while meeting the constraints of shot size, clamping force, and stroke length. In TABLE 1 we compare the results for our part with the specifications of our selected machine[23].

TABLE 1: COMPARISON OF ESTIMATED CLAMPING FORCE, SHOT SIZE, AND STROKE LENGTH FOR THE PART ALONG WITH MAXIMUM POSSIBLE VALUES FOR THE CLOSEST-MATCH INJECTION MOLDING MACHINE.

\begin{tabular}{lll}
\hline Parameter & Experimental Part & 5.5kW Machine \\
$F_{\text {clamp }}$ & $104 \mathrm{kN}$ & $300 \mathrm{kN}$ \\
$V_{\text {cavity }}$ & $20.87 \mathrm{~cm}^{3}$ & $34 \mathrm{~cm}^{3}$ \\
$L_{\text {stroke }}$ & $6.080 \mathrm{~cm}$ & $20 \mathrm{~cm}$ \\
\hline
\end{tabular}

\section{ESTIMATION OF CYCLE TIMES}

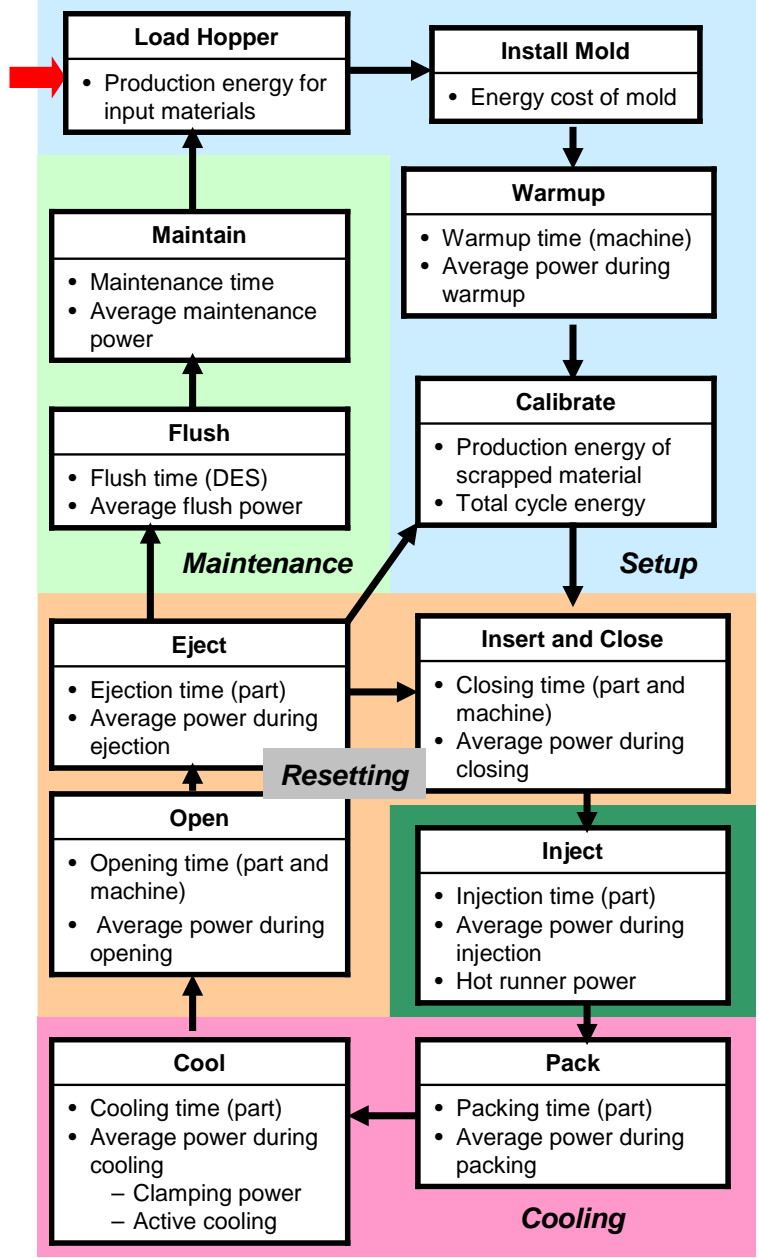

FIGURE 9: STATE-TRANSITION DIAGRAM OF A
TYPICAL INJECTION-MOLDING OPERATION.

Once the machine has been selected, the cycle time for the part can be estimated. The molding cycle can be broken down into three stages: injection, packing and cooling, and reset. These stages, as well as their sub-stages and other auxiliary stages in a typical injection molding operation, are shown in the state transition diagram in FIGURE 9.

During the injection stage, the pressure at the injection nozzle is gradually increased. This is done to maintain a constant volumetric flow rate, as the melt cools and solidifies. The estimated fill time for the mold cavity can be derived based on the maximum flow rate [4]. This relationship is as follows:

$t_{\text {fill }}=\frac{2 V_{\text {cavity }}}{Q_{\max }}$

where $Q_{\max }$ is the maximum flow rate of polymer from the nozzle. 
Next, the pressure is held and then gradually dropped as the part cools and contracts in the mold. We assume that active cooling is not used. Using the first term of the Carslaw and Jaeger solution [24], the cooling time in seconds can be estimated from the maximum wall thickness of the part and the processing parameters and thermal diffusivity of the polymer. The maximum wall thickness can be determined from the part model, and the processing parameters can be found from the material datasheet provided by the supplier. Given:

$h_{\max } \quad$ maximum wall thickness of part

$T_{i} \quad$ polymer injection temperature

$T_{m} \quad$ recommended mold temperature

$T_{x} \quad$ recommended part ejection temperature

$\alpha \quad$ thermal diffusivity of material

We can estimate the cooling time as:

$t_{\text {cool }}=\frac{h_{\max }^{2}}{\pi^{2} \alpha} \ln \left(\frac{4\left(T_{i}-T_{m}\right)}{\pi\left(T_{x}-T_{m}\right)}\right)$

The thermal diffusivity can be computed from the specific heat, thermal conductivity, and density of the material as:

$\alpha=\frac{k}{\rho_{\text {resin }} c_{\text {resin }}}$

where $k$ is the thermal conductivity of the material. Finally, after ejection of the part, the mold is prepared for the next cycle. This time is estimated by applying an overhead to the dry cycle time for the machine. The dry cycle time is a performance metric that indicates the time for the machine to perform the actions necessary to manufacture a part, without the part actually being produced. The overhead is derived from the part depth $(D)$ and stroke length $\left(L_{\text {stroke }}\right)$. Adding a 1-second dwell ejection, the reset time is calculated as:

$t_{\text {reset }}=1+\left(1.75 \sqrt{\frac{L_{\text {stroke }}}{L_{\text {stroke }}^{\text {max }}}}\right) t_{d}$

where $t_{d}$ is the dry cycle time for the machine.

For our example, we use the machine that we selected in the previous section. This is the least expensive machine capable of producing our part. The machine parameters are given in TABLE 2. The material properties for Hival ABS HG6 Natural are provided in TABLE 3.

TABLE 2: TABLE OF PARAMETERS FOR THE SELECTED INJECTION MOLDING MACHINE.

Parameter $5.5 \mathrm{~kW}$ Machine

\begin{tabular}{ll}
$Q_{\max }$ & $55 \mathrm{~cm}^{3} / \mathrm{s}$ \\
$t_{d}$ & $1.7 \mathrm{~s}$ \\
$C_{\text {machine }}$ & $28 \$ \mathrm{US} / \mathrm{hr}$ \\
\hline
\end{tabular}

TABLE 3: TABLE OF PARAMETERS FOR THE SELECTED MATERIAL.

\begin{tabular}{llll}
\hline Param. & Hival ABS & Param. & Hival ABS \\
$C_{\text {resin }}$ & $1.96 \mathrm{~J} / \mathrm{g}^{\circ} \mathrm{C}$ & $T_{i}$ & $240{ }^{\circ} \mathrm{C}$ \\
$\rho_{\text {resin }}$ & $1.04 \mathrm{~g} / \mathrm{cm}^{3}$ & $T_{m}$ & $50{ }^{\circ} \mathrm{C}$ \\
$\alpha$ & $.0009272 \mathrm{~cm}^{2} / \mathrm{s}$ & $T_{x}$ & $109{ }^{\circ} \mathrm{C}$ \\
\hline
\end{tabular}

Given these values, we can compute the cycle times as follows:

$t_{\text {fill }}=0.7589 \mathrm{~s}$

$t_{\text {cool }}=12.74 \mathrm{~s}$

$t_{\text {reset }}=2.640 \mathrm{~s}$

\section{ESTIMATION OF SETUP OPERATIONS}

For our application, we seek to determine the amount of energy consumed during machine setup, per part. This is done by determining the total energy used during the machine setup before the start of the production. Setup processes include steps such as warming up the machine, installing the mold, and calibrating the machine. The injection molding machine consumes significant amount of energy during warmup, and then continues to consume energy as it idles during mold installation. Before start of production, the injection molding process needs to be stabilized. This is done to establish process equilibrium to ensure complete filling of the part, avoid jetting etc. Manufacturers typically reject the first few tens of parts before beginning the production. We therefore include the energy consumed during this step as part of the machine calibration.

To determine the total energy used during setup processes, we must first determine how often the machine must be set up during the production schedule of the entire production volume. Typically, the entire production volume will not be completed in a single production run. Typical injection molded parts are produced based on the production requirement and the delivery schedule. The customer specified delivery schedule involves a request for a certain number of parts at regular time intervals. Thus, to save on the inventory cost before delivery to the customer, the manufacturer makes parts in batches. The batch size should be larger than the number of parts delivery requirement at each time interval. Therefore, any remaining parts must be stored at the expense of the manufacturer until the next delivery. However, larger batch sizes require fewer setups. Therefore, there is a tradeoff between the setup cost and the inventory cost.

FIGURE 10 shows the relationship between the delivery schedule and the production schedule over the entire production volume. The manufacturer produces a certain number of parts, and delivers to the customer at regular 
intervals. During this time, undelivered parts remain in storage. When the parts in storage have been depleted, the manufacturer makes a new batch of parts, and continues to ship them out according to the customer's delivery schedule. We assume a regular delivery interval for our purposes.

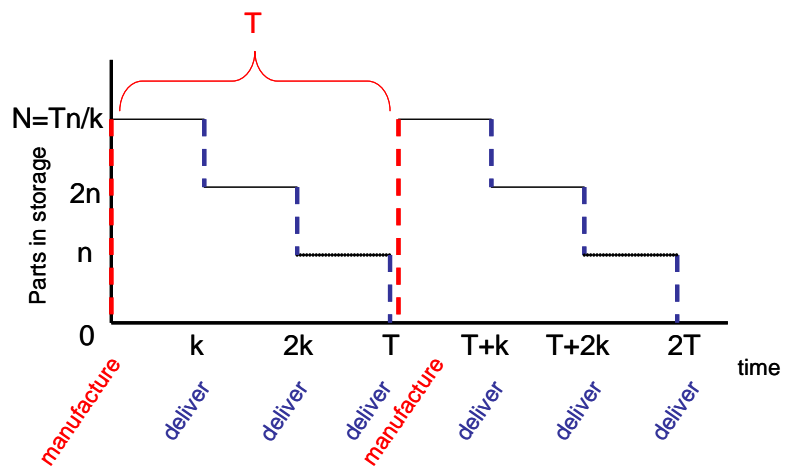

FIGURE 10: GRAPH SHOWING DELIVERY SCHEDULE AND PRODUCTION SCHEDULE IN TERMS OF PARTS IN STORAGE VERSUS TIME.

This tradeoff can be formulated as a single variable optimization problem. The solution to this problem gives us the optimal number of setup operations which minimize the cost to the manufacturer over the entire production volume. For this problem, we assume that the batch production period is much larger than the delivery period, and so lead time can be ignored. Furthermore, we assume that the manufacturer must pay for a constant amount of storage; even as the manufacturer's inventory is depleted, they must continue to pay for the entire space needed to accommodate a batch of parts. Given:

We formulate our optimization problem as follows.

$\begin{array}{ll}N & \text { batch production volume } \\ X & \text { total production volume } \\ n & \text { delivery volume } \\ k & \text { delivery period (days) } \\ C_{\text {setup }} & \text { cost to set up one batch } \\ c_{\text {store }} & \text { cost to store one unit per day } \\ T & \text { manufacturing period (days) } \\ q_{\text {setups }}=X k / T n & \text { number of setup operations } \\ q_{\text {store }}=X T & \text { storage quantity in item-days }\end{array}$

where $T$ is our design variable, we can minimize the total cost $C(T)$ as follows:

$\min _{T} C(T)=q_{\text {setups }} c_{\text {setup }}+q_{\text {store }} C_{\text {store }}$

s.t.

$T>k>0$

Making substitutions for $q_{\text {setups }}$ and $q_{\text {store }}$, we get
$C(T)=\frac{X k}{T n} c_{\text {setup }}+X T c_{\text {store }}$

Using KKT conditions, we arrive at the following solution:

$T=\sqrt{\frac{k c_{\text {setup }}}{n c_{\text {store }}}}$

Thus we can determine the optimal number of setup operations which minimize cost as:

$q_{\text {setups }}=\frac{X k}{T n}$

For our example, we assume the total production volume, delivery volume, and delivery period as:

$X=2000000$

$n=50000$

$k=14$ days

Furthermore, we assume that the cost of a single setup operation is proportional to the setup time and the hourly machine rate; i.e.:

$C_{\text {setup }}=C_{\text {machine }} t_{\text {setup }}$

$C_{\text {setup }}=56$ \$US

To determine the storage cost, we used the average rate for public storage as advertised by Public Storage [25]. A typical 10'x10'x8' storage space in the College Park area costs approximately 150 \$US per month. Assuming a 25\% packing ratio, this is equivalent to:

$C_{\text {store }}=8.8287 \times 10^{-7}$ SUS per $\mathrm{cm}^{3}$ per day.

Thus, computing the optimal batch period gives us:

$T \approx 133$ days

and therefore:

$q_{\text {setups }} \approx 4$

In other words, we will make 500,000 parts at a time.

\section{ESTIMATION OF TOTAL ENERGY CONSUMPTION}

The energy used during filling, cooling, and resetting can be determined from the cycle times and the power profile of the machine. We have already determined the cycle time of the part, including the times required to fill the mold, cool the part, and reset the machine. Gutowski [9] and Krishnan [10,11] have published energy consumption profiles for various injection molding machines. We assume that energy consumption per unit of time on a given machine is constant for a given part of the cycle. Therefore, we can look up the power required, in watts, for the machine during each stage of the injection molding cycle. Given: 
Power $_{\text {fill }}$

avg. power used to fill the mold, $\mathrm{kW}$

Power $_{\text {cool }}$ avg. power used to cool the part, $\mathrm{kW}$

Power avg. power used to reset the machine, $\mathrm{kW}$

We can determine in kilojoules the energy used during filling, $E_{\text {fill }}$, the energy used during cooling, $E_{\text {cool }}$, and the energy used to reset the machine, $E_{\text {reset }}$ as:

$$
\begin{aligned}
& E_{\text {fill }}=\frac{\operatorname{Power}_{\text {fill }} t_{\text {fill }}}{n_{\text {cavities }}} \\
& E_{\text {cool }}=\frac{\text { Power }_{\text {cool }} t_{\text {cool }}}{n_{\text {cavities }}} \\
& E_{\text {reset }}=\frac{P o w e r_{\text {reset }} t_{\text {reset }}}{n_{\text {cavities }}}
\end{aligned}
$$

To determine the average powers shown in TABLE 4, we measured the power consumption on a $2.9 \mathrm{~kW}$ Milacron Babyplast injection molding machine available in our lab. This was done by connecting a clamp-on multimeter to the threephase power supply of the Milacron Babyplast. We then warmed up the machine, and molded several sample parts. We recorded the average power consumption using an Extech meter during warmup and during the three major stages of molding. These measurements are shown in TABLE 4. We calculated the expected power consumption for the selected machine by scaling the measured powers for the Milacron Babyplast injection molding machine by the ratio of its driving power of $2.9 \mathrm{~kW}$, to the driving power of the $5.5 \mathrm{~kW}$ machine. In the future, we plan to directly instrument a wide variety of injection molding machines to obtain a more accurate scaling law for average power consumption.

TABLE 4: AVERAGE POWER CONSUMPTION OF EACH STAGE OF MOLDING CYCLE FOR SELECTED MACHINE.

\begin{tabular}{lll}
\hline Parameter & Babyplast & $\mathbf{5 . 5} \mathbf{~ k W ~ M}$ \\
Power $_{\text {setup }}$ & $0.8823 \mathrm{~kW}$ & $1.673 \mathrm{~kW}$ \\
Power $_{\text {fill }}$ & $1.6 \mathrm{~kW}$ & $3.034 \mathrm{~kW}$ \\
Power $_{\text {cool }}$ & $0.744 \mathrm{~kW}$ & $1.411 \mathrm{~kW}$ \\
Power $_{\text {reset }}$ & $1.265 \mathrm{~kW}$ & $2.399 \mathrm{~kW}$
\end{tabular}

Using these values, we find a result of:

$$
\begin{aligned}
& E_{\text {fill }}=0.5756 \mathrm{~kJ} \\
& E_{\text {cool }}=4.4927 \mathrm{~kJ} \\
& E_{\text {reset }}=1.583 \mathrm{~kJ}
\end{aligned}
$$

The other quantities we must calculate are the amounts of energy used during setup and calibrating the machine. Assuming that we also know the average power in kilowatts used by the machine during setup, Power $_{\text {setup }}$, and the average time required to setup the machine in seconds, $t_{\text {setup }}$, we can determine the energy used during a setup operation, multiply it by the number of setup operations, and divide by the total production volume. Thus, we allocate the total setup energy to arrive at the per-part setup energy as:

$E_{\text {setup }}=$ Power $_{\text {setup }} t_{\text {setup }}\left(\frac{q_{\text {setups }}}{X}\right)$

For our operation, we assume that the warm up time for each machine set up is two hours, or 7200 seconds. This gives us the setup energy of:

$E_{\text {setup }}=0.0241 \mathrm{~kJ}$

Next, we allocate the energy used to mold each part during calibration, to the total production volume. Assuming that $x_{\text {calibrate }}$ parts are made and discarded during the calibration process, we arrive at the per-part calibration energy as:

$E_{\text {calibrate }}=\left(E_{\text {fill }}+E_{\text {cool }}+E_{\text {reset }}\right)\left(\frac{q_{\text {setups }} x_{\text {calibrate }}}{q_{\text {setups }} x_{\text {calibrate }}+X}\right)$

For our operation, we assume:

$X_{\text {calibrate }}=250$ parts

Thus, we can compute $E_{\text {calibrate }}$ as follows:

$E_{\text {calibrate }}=\left(E_{\text {fill }}+E_{\text {cool }}+E_{\text {reset }}\right) / 2001$

$E_{\text {calibrate }}=0.0033 \mathrm{~kJ}$

Adding up these energies, we get the total energy consumed per good part produced as:

$E=E_{\text {setup }}+E_{\text {calibrate }}+E_{\text {fill }}+E_{\text {cool }}+E_{\text {reset }}$

The estimated total energy consumption for our part is:

$E=6.679 \mathrm{~kJ}$

It is clear that cooling and resetting dominate the energy consumption for our example part. Although the filling stage uses the most power, filling happens very quickly and thus does not dominate the energy used. Setup and calibration have also been shown to have small, but measurable contributions to energy consumption.

At this point, we have only estimated the energy consumed during injection molding. For a complete life cycle analysis, we would need to determine the energy consumed during production of the polymer materials, the energy used for transportation during the various stages in the supply chain, energy associated with the part's usage, and energy consumed during disposal. This work focuses only on manufacturing, and so the other stages of the product life cycle were not addressed. 


\section{CONCLUSION}

This paper is the first attempt at developing a methodology for obtaining an accurate estimate of the total energy consumption for production of injection molded parts by incorporating the different aspects of the molding cycle. This methodology can be applied at the design stage, and thus allows the designer to make energy-conscientious decisions before the part goes into production. We present a method for estimating the energy consumption by 1) selecting the runner layout based on part similarities, 2) performing physics based simulations on the specific part to first select the machine for injection molding and then estimate the cycle time for production, 3) computing the production volume based on the delivery schedule, the energy overheads (machine setup energy, calibration energy etc.) for each production run and the inventory cost, and 4) estimating the total energy usage using by performing physical experiments to measure the power profiles on an injection molding machine. Finally, multiplying these times with the average power consumed during each stage of the process, and adding up the results, gives us the total perpart energy consumption.

In future work, we plan to test the validity of our model by using other parts on different machines and measuring the actual energy consumption on those machines. We hope that a more accurate model of energy consumption for molding plastic parts will help designers make better, more environmentally-conscientious decisions during the design process, rather than waiting until manufacturing has already begun to perform energy consumption audits.

Acknowledgements: This research is supported in part by the National Institute of Standards and Technology's (NIST) Manufacturing System Integration Division.

\section{REFERENCES}

1. Plastemart. Chinese injection molding industry is poised for a good growth. 2009; Available from: http://www.plastemart.com/upload/Literature/Injection -molding-industry-China-poised-for-goodgrowth\%20.asp.

2. EIA International Energy Annual 2006. 2006, US Energy Information Administration.

3. EDGAR 3.2 Fast Track 2000. 2000, Netherlands Environmental Assessment Agency.

4. Boothroyd, G., P. Dewhurst, and W. Knight, Product Design for Manufacture and Assembly. 2 ed. 2002, New York: Marcel Dekker.

5. Jolliet, O., et al., IMPACT 2002+: A New Life Cycle Impact Assessment Methodology. INTERNATIONAL JOURNAL OF LIFE CYCLE ASSESSMENT, 2003. 10(6): p. 324-330.

6. PE_International, GaBi 4. 2008: LeinfeldenEchterdingen, Germany
7. PRé_Consultants, SimaPro 7.1. 2006: Amersfoort, The Netherlands.

8. Thiriez, A. and T. Gutowski, An Environmental Analysis of Injection Molding, in ISEE. 2006.

9. Gutowski, T., J. Dahmus, and A. Thiriez, Electrical Energy Requirements for Manufacturing Processes, in $13^{\text {th }}$ CIRP International Conference on Life Cycle Engineering. 2006: Leuven, Belgium.

10. Krishnan, S.S., et al., Machine Level Energy Efficiency Analysis in Discrete Manufacturing for a Sustainable Energy Infrastructure, in International Conference on Infrastructure Systems 2009. 2009: Chennai, India.

11. Krishnan, S.S., et al., Sustainability Analysis and Energy footprint based Design in the Product Lifecycle, in Indo-US Workshop on Designing Sustainable Products, Services and Manufacturing Systems,. 2009: Bangalore, India.

12. Morrow, W.R., et al., Environmental aspects of laserbased and conventional tool and die manufacturing. Journal of Cleaner Production, 2007. 15: p. 932-943.

13. Beaumont, J.P., Runner and Gating Design Handbook: Tools for Successful Injection Molding. 2004: Hanser Gardner Publications

14. Cheng, J., et al., Optimization of injection mold based on fuzzy moldability evaluation. Journal of Materials Processing Technology, 2008. 208(1-3): p. 222-228.

15. Zhai, M., Y. Lam, and C. Au, Runner sizing in multiple cavity injection mould by non-dominated sorting genetic algorithm. Engineering with Computers, 2009. 25(3): p. 237-245.

16. Li, C.S. and Y.K. Shen, Optimum design of runner system balancing in injection molding. International Communications in Heat and Mass Transfer, 1995. 22(2): p. 179-188.

17. Alam, K. and M.R. Kamal, A robust optimization of injection molding runner balancing. Computers \& Chemical Engineering, 2005. 29(9): p. 1934-1944.

18. Zhai, M., Y. Lam, and C. Au, Runner sizing and weld line positioning for plastics injection moulding with multiple gates. Engineering with Computers, 2006. 21(3): p. 218-224.

19. Lam, Y.C. and L.W. Seow, Cavity balance for plastic injection molding. Polymer Engineering \& Science, 2000. 40(6): p. 1273-1280.

20. Ashland. Ashland Distribution. 2010; Available from: www.ashland.com.

21. Moldflow, Moldflow Plastics Insight 6.1. 2009: Framingham, Massachusetts.

22. Custompart.net: Free Online Manufacturing Cost Estimation and Education Resource. 2008; Available from: http://www.custompartnet.com.

23. Babyplast, Babyplast Micro Injection Molding Machines. 2010.

24. Carslaw, H.S. and J.C. Jaeger, Conduction of Heat in Solids. 1986, Oxford: Clarendon Press.

25. PS. Public Storage. 2010; Available from: www.publicstorage.com. 
